\title{
Enhanced Slack Time based Price Driven Demand Response for Future Effectual Smart Communities
}

\author{
Bhagya Nathali Silva, Murad Khan, Kijun Han
}

\begin{abstract}
Evolution of smart grid concept aims to address the imbalance between electricity demand and supply. Owing to consideration on sustainable energy, user comfort, and cost efficiency, residential Demand Response (DR) has gained a remarkable popularity over the past few years. To further enhance these benefits, herein we propose a residential appliance scheduling algorithm inspired by Least Slack Time (LST) algorithm. The conventional LST algorithm is amended with consumption thresholds and waiting factor constraints to derive proposed Minimum Slack Time (MST) algorithm, which increase cost and comfort efficiency during DR. Proposed algorithm was experimented in a simulated residential community consists of 50 houses. Further experiments were conducted by aggregating renewable energy sources using aggregated MST (AMST) algorithm. All instances were compared with an existing scheduling mechanism to assure superiority of proposed MST and AMST algorithms, in terms of grid electricity consumption, cost, Peak-to-Average Ratio (PAR), and waiting time.
\end{abstract}

Keywords: Cost efficient scheduling, Minimum slack time, Peak load reduction, Residential demand response, User convenience

\section{INTRODUCTION}

Conventional grids were evolved into smart grids with the advancements in communication technologies, energy storages, renewable energy sources, and Advanced Metering Infrastructures (AMI) [1, 2]. Although smart grids have promoted cost reduction, demand management, and renewable energy utilization, unceasingly escalating energy demand remains as a critical challenge in the modern world [3]. Hence, global attention was drawn towards small-scale power plants embed with renewable energy generators e.g. photovoltaic (PV) panels and wind turbines [4]. Moreover, Demand Response (DR) programs were introduced at the demand end to efficiently manage energy usage patterns. Adjustments in usage patterns aim to obtain cost benefits,

Revised Manuscript Received on December 30, 2019.

* Correspondence Author

Bhagya Nathali Silva, School of Computer Science and Engineering, Kyungpook National University, Daegu, Korea.

Murad Khan, School of Computer Science and Engineering, Kyungpook National University, Daegu, Korea.

Kijun Han*, School of Computer Science and Engineering, Kyungpook National University, Daegu, Korea.

(C) The Authors. Published by Blue Eyes Intelligence Engineering and Sciences Publication (BEIESP). This is an open access article under the CC BY-NC-ND license (http://creativecommons.org/licenses/by-nc-nd/4.0/) while maintaining the balance between electricity demand and supply [5-8]. DR programs alter energy consumption behaviors of users to maximize cost efficiency and renewable energy utilization, meanwhile reducing the dependency on grid electricity $[9,10]$. DR programs are in two types, namely incentive driven and price driven. Incentive driven programs offer incentives to consumers considering their load adjustments. Whereas in price driven DR, consumers are enforced to get the maximum benefit from time varying tariffs. Price driven DR aims to reduce overall energy consumption and cost by shifting peak loads to off peak hours, in order to maximize utilization of periods with lower tariffs. Time of Use (ToU), Real-Time Pricing (RTP), Critical Peak Pricing (CPP), and Inclined Block Rate (IBR) pricing are some widely used price driven DR approaches.

Recently, a lot of insightful works on price drive DR have been proposed to encourage optimal utilization of lower tariffs. Nevertheless, a majority of these works were proposed for single house scenarios [10-17]. A greedy method based appliance scheduler that incorporates neural networks with multiple energy sources has been proposed by Shukla et al. [14] to improve energy efficiency. Multiple schemes for autonomous appliance scheduling based on price driven DR were proposed by Khan et al. and Silva et al., in order to preserve energy, while reducing cost on energy [15-17]. Muratori et al. proposed a multi-ToU based dynamic domestic energy management approach to obtain a flat demand for a particular day [8]. Another price driven DR program for residential community was proposed in [18] using a mutation operator integrated ant colony optimization algorithm. Zhao et al. proposed a domestic DR program using IBR and RTP to avoid new peak formation problem arise with RTP based DR [19]. A task priority based appliance scheduling scheme was proposed by Rastegar et al. incorporating Value on Lost Load (VOLL) mechanism [20]. This mechanism schedules tasks according to given task priorities and allows forceful termination of low priority tasks, in order to maintain demand curve. Regardless of valuable contributions from previous works, still a larger room remains for research in DR in real-world aspect, due to unceasing growth in energy demand, depletion of non-renewable energy sources, increasing carbon emission, and volatility of energy cost.Herein, we propose a Least Slack Time (LST) inspired Minimum Slack Time (MST) based appliance scheduling algorithm incorporating price driven DR to manage residential electricity demand.

Although many DR programs

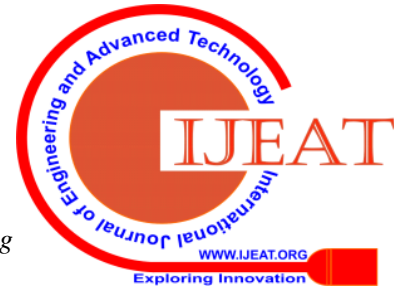


have been proposed during last few years, a majority of these works focused on single house scenarios, which restrict the implementation feasibility in real world context. Hence, the proposed algorithm's performance was evaluated for a multiple houses context occupied by multiple users. The utmost goals of the proposed work are to minimize grid electricity consumption and cost, while maximizing user comfort and renewable energy usage. Proposed algorithm performance was evaluated for two instances. MST instance evaluates the algorithm without aggregating renewable energy sources. Whereas aggregated MST (AMST) evaluates the performance of same algorithm by additionally introducing renewable energy sources. Performance evaluations were compared with a no scheduling (NS) instance and a VOLL scheduling instance proposed by Rastegar et al. VOLL scheduling was selected considering its operational similarity to proposed algorithm, since both algorithms operate according to dynamic priorities. As the breakthrough of the MST and AMST, consumption thresholds, waiting factor, and renewable energy sources were introduced to the conventional LST algorithm. Results obtained from experiments reveal the superiority of proposed algorithm and thus, we can claim introduced amendments to the LST led MST and AMST towards remarkable performance improvements in domestic energy management tasks.

\section{METHODOLOGY}

\section{A. Overview}

Energy usage behaviors of users are uncertain and as a result energy management in real-world context remains challenging. Hence, experts in both academia and industry aim to fine-tune energy management strategies such as DR to reduce energy wastage, cost, grid energy utilization, and peak demand, while preserving user comfort. In order to achieve these goals, this article proposes a price driven DR approach based on LST algorithm. Similar with conventional LST, proposed MST algorithm schedules energy related tasks considering the slack time of a task. Slack time is the time difference between desired deadline and actual deadline, if a task starts its operation right at this moment. Fig. 1 clearly presents the definition of slack time. Proposed MST algorithm prioritize tasks in ascending order of slack time. In other words, highest priority will be given the tasks with least slack time. MST inherits its characteristics from conventional LST algorithm. Hence, MST becomes highly potential for scheduling uncertain tasks such as users' energy requests, since it does not consider prior assumptions on task occurrence rate. Appliance load profiles and user requests influence electricity consumption patterns of a household. Therefore, an effective appliance scheduling program should essentially consider load profiles of appliances and user requests. In proposed MST scheduler, three types of load shift models were considered namely, generic shifting model (GSM), flexible shifting model (FSM), and periodic shifting model (PSM), which are elaborately discussed in one of our previous works [16]. GSM consists of appliances with partial flexibility, whereas appliances with additional flexibility belong to FSM. PSM is applied on appliances with periodic operational load profiles.

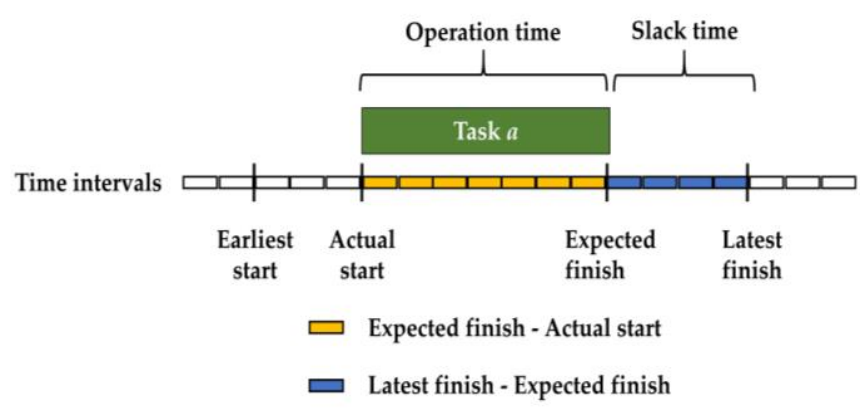

Fig. 1. Overview of task slack time

In proposed scenario all houses in the residential community are equipped with a smart meter that facilitates bidirectional communication between users and utility, PV panels and an ESS. MST scheduler is aware about all appliances and sensors deployed in the house and disclose any required information to the grid.

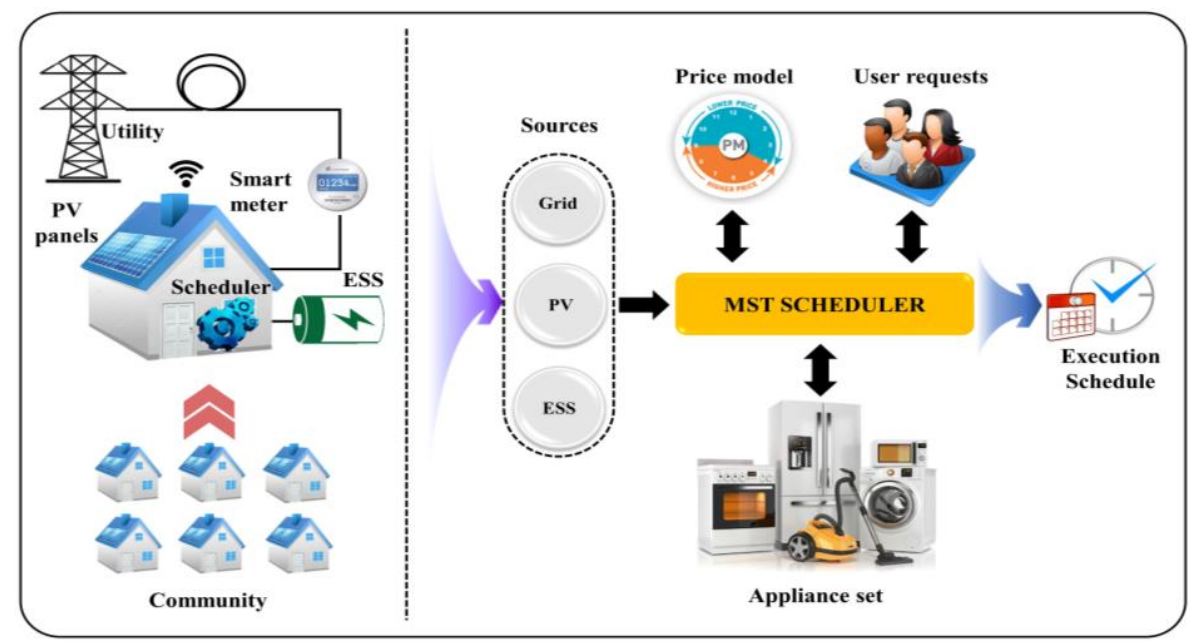

Fig. 2. Overview of the proposed MST scheduling system for the residential community 


\section{B. MST Scheduler}

The experimented residential community $(X)$ consists of 50 houses $(x)$, where $x \in X, X=\left\{x_{1}, x_{2}, x_{3}, \ldots, x_{50}\right\}$. Each house consists of ten appliances $(a)$ and total appliance set for the community is given by $A$, where $a \in A, A=\left\{a_{1}, a_{2}, a_{3}, \ldots, a_{500}\right\}$. Further, each house resides four consumers $(c)$ and total consumer set of the community is given by $C$, where $c \in C, C=\left\{c_{1}, c_{2}, c_{3}, \ldots, c_{200}\right\}$. Operation parameters namely earliest start $e s_{a}$, latest finish $l f_{a}$, task duration $t d_{a}$, and power consumption $p c_{a}$ are defined by the consumer according to desired comfort measures and electricity tariffs. ToU pricing is used as the price driven DR mechanism to calculate electricity bill. Grid electricity, PV panels, and ESS are connected to the MST scheduler to optimize energy utilization. As illustrated in Fig. 2, ToU tariff from utility and user requests are the inputs to the MST scheduler. Accordingly, MST scheduler determines the operational schedule for residential energy tasks using MST algorithm.

The MST algorithm is executed for a duration of 24 hours. Total duration ( $T$ ) is subdivided to intervals $(t)$ of one hour, where $t \in T, T=\left\{t_{1}, t_{2}, t_{3}, \ldots, t_{24}\right\}$. Each time interval belongs strictly to a single demand phase $\left(\alpha_{x}\right)$. Demand phase can be either off-peak ( $\left.\alpha_{1}\right)$, average-peak ( $\left.\alpha_{2}\right)$, or peak $\left(\alpha_{3}\right)$ phases. Demand phase for each $t$ interval is given as below.

$$
\alpha_{x}\left\{\begin{array}{llll}
\alpha_{1}, & 0 \leq t<6 & \text { OR } & 22 \leq t<24 \\
\alpha_{2}, & 6 \leq t<10 & \text { OR } & 20 \leq t<22 \\
\alpha_{3}, & 10 \leq t<20 &
\end{array}\right.
$$

In price driven DR, electricity bill calculation depends on demand phase and corresponding energy consumption. MST algorithm incorporated ToU based DR. ToU defines energy cost per unit $\left(R_{x}\right)$ respective to the demand phase as below.

$$
R_{x} \begin{cases}R_{1}, & t \in \alpha_{1} \\ R_{2}, & t \in \alpha_{2} \\ R_{3}, & t \in \alpha_{3}\end{cases}
$$

In proposed MST, consumption threshold ( $\left.\theta_{x}\right)$ is defined for each demand phase and these thresholds contribute to the performance improvement in terms of reducing grid electricity utilization and increasing renewable energy utilization. Further, consumption thresholds distinguish MST from conventional LST algorithm. Consumption thresholds for each demand phase is given as below.

$$
\theta_{x} \begin{cases}\theta_{1}, & t \in \alpha_{1} \\ \theta_{2}, & t \in \alpha_{2} \\ \theta_{3}, & t \in \alpha_{3}\end{cases}
$$

Let $E_{a}$ denotes energy consumption of appliance $a$ for a single operation cycle. Energy consumption of appliance $a$ during $t$ and energy consumption of all appliances during $t$ are denoted by $E_{a t}$ and $E_{A t}$ respectively. Accordingly, energy consumption of $a$ during total period and energy consumption of all appliances during total period are denoted by $E_{a T}$ and $E_{A T}$ as defined below.

$$
\begin{aligned}
& E_{a}=p c_{a} \bullet t d_{a} \\
& E_{a t}=\sum_{t} E_{a} \\
& E_{A t}=\sum_{a=1}^{A} E_{a t} \\
& E_{a T}=\sum_{t=1}^{T} E_{a t} \\
& E_{A T}=\sum_{t=1}^{T} E_{A t}
\end{aligned}
$$

Let $\Pi_{a}$ determines cost of a single operational task. $\Pi_{a t}, \Pi_{A t}, \Pi_{a T}$, and $\Pi_{A T}$ calculates energy costs respective to $E_{a t}, E_{A t}, E_{a T}$, and $E_{A T}$.

$\Pi_{a}=R_{x} \cdot E_{a}$

$\Pi_{a t}=\sum_{t} \Pi_{a}$

$\Pi_{A t}=\sum_{a=1}^{A} \Pi_{a t}$

$\Pi_{a T}=\sum_{t=1}^{T} \Pi_{a t}$

$\Pi_{A T}=\sum_{t=1}^{T} \Pi_{A t}$

AMST facilitates alternative energy utilization through PV panels or ESS. PV panels are used to charge ESS or to operate domestic appliances during peak hours. Although PV energy generation depends on the angle of the sunlight, herein we considered only the duration with direct sunlight. The proposed work assume that duration of direct sunlight $(D)$ is 5 hours. PV energy duration during $t$ interval is denoted by $\Psi_{t}$ and determined by rated generation capacity of a PV panel $\left(P_{P V}\right)$ and number of PV panels $\left(N_{P V}\right)$. Let $E_{P V}$ denotes total energy generation for experimented period, when $D$ is total duration with direct sunlight and $t \in D$.

$\Psi_{t}=P_{P V} \cdot N_{P V}$

$E_{P V}=\Psi_{t} \cdot D$

In all instances, ESS status ( $E S S_{\theta}$ ) is either charging (1) or discharging (0) and represented as a Boolean variable. Flow constraints and capacity constraints are defined for both statuses. Let $E S S_{r, \max }^{c}$ and $E S S_{r, \max }^{d}$ denote maximum charging and discharging flow rates. Capacity constraints regulate maximum capacity $\left(E S S_{\max }\right.$ ) during charging and minimum capacity ( $E S S_{\text {min }}$ ) during discharging, when $\triangle E S S$ denotes current storage level of the ESS.

$$
\begin{aligned}
& E S_{\theta}\left\{\begin{array}{l}
1, \text { Charging } \\
0, \text { Discharging }
\end{array}\right. \\
& E S S=1, \Delta E S S<E S S_{\text {max }} \text { AND ESS } S_{r}^{c}<E S S_{r, \text { max }}^{c} \\
& E S S=0, \Delta E S S>E S S_{\text {min }} \text { AND ESS } S_{r}^{d}<E S S_{r, \text { max }}^{d}
\end{aligned}
$$


Reducing PAR value is another objective proposed MST. PAR value reduction depends on energy consumption of all houses in the residential community. Hence, $P A R_{\text {com }}$ is defined as below for the community, when $E_{t, x}$ denotes maximum energy utilization of $x^{\text {th }}$ house during $t$ time.

$$
P A R_{\text {com }}=\frac{\max \left(E_{t, x}\right)}{\frac{1}{T}\left[\sum_{x=1}^{X}\left[\sum_{t=1}^{T} E_{t, x}\right]\right]}
$$

In contrasting to existing works that do not quantifies user convenience during scheduling, proposed work quantitatively determines waiting time according to user preference. Waiting time $\left(w_{a}\right)$ is calculated from maximum desired waiting time $\left(\max _{w}\right)$ defined by users and waiting factor $\left(\omega_{f}\right)$. The minimal waiting time is determined by considering $\max _{w}, \omega_{f}$, and expected operation period $\left(\beta_{a}\right)$ of a task, when $\beta_{a} \in\left(e s_{a}, l f_{a}-t d_{a}\right)$. Let $\omega_{f}$ is a real value between zero and one.

$$
\begin{aligned}
& \omega_{f}=\frac{\beta_{a}-e s_{a}}{l f_{a}-t d_{a}-e s_{a}} \\
& w_{a}=\omega_{f} \cdot \max _{w}
\end{aligned}
$$

MST algorithm defines the objective function as a minimization function of cost and waiting factor. Cost of grid electricity, cost of uninterrupted frame allocation, and waiting factor are the optimization variables. Below given objective function was evaluated using mixed integer linear programming (MILP).

$$
\min \sum_{t=1}^{T}\left[\sigma_{1} \cdot \sum_{a=1}^{A}\left(\Pi_{a} \cdot \rho_{a}\right)+\sigma_{1} \cdot \sum_{a=1}^{A}\left(\Pi_{\eta} \cdot \eta_{a}\right)\right]+\sigma_{2} \bullet \omega_{f}
$$

Inverse correlation between cost and comfort is demonstrated using user defined cost priority constant $\left(\sigma_{1}\right)$ and comfort priority constant $\left(\sigma_{2}\right)$, where $\sigma_{1}+\sigma_{2}=1$. Appliance status $\left(\rho_{a}\right)$ can be either on (1) or off (0). Requirement for uninterrupted frame allocation comes with a cost and denoted by $\eta_{a}$, where $\eta_{a}=1$ requires continuous frames and $\eta_{a}=0$ is otherwise as per to user demand.

\section{Experiment Setting}

The community energy management system was simulated on Visual Studio 2016 on .NET framework 4.6 using Visual C\# language. The community consists of 50 houses, where each house resides four consumers and deploys ten appliances. Appliances belong to GSM, FSM, and PSM shifting profiles. Refrigerator and light bulbs are considered as non-schedulable appliances. Uncertain behavior of consumers is replicated in the experiment setting using a random variable to initiate consumer requests. Simulation period was 24 hours divided in to off-peak, average-peak, and peak hours. Simulation was simultaneously executed for NS, VOLL, MST, and AMST instances to maintain consistency of consumer requests and energy demand. Grid energy allocated for the community per hour is $2500 \mathrm{kWh}$. In AMST instance, ESS and PV panels are integrated to the house. Each house installs ten PV panels with $250 \mathrm{~W}$ rated energy to cover $16.35 \mathrm{~m}^{2}$ roof area ( 1 panel $=65$ " x 39") and generates solar power with direct sunlight for a period of six hours. Each ESS stores $10 \mathrm{kWh}$ with a $90 \%$ depth of discharge (DoD). ToU tariffs corresponding to each demand phase are given in below Table I.

Table I. ToU tariffs corresponding to demand phases and operational intervals

\begin{tabular}{|c|c|c|}
\hline Demand phase $\left(\alpha_{x}\right)$ & Interval $(t)$ & Tariff (cents) \\
\hline \multirow{2}{*}{$\alpha_{1}$} & $0 \leq t<6$ & \multirow{2}{*}{5.5} \\
\cline { 2 - 2 } & $22 \leq t<24$ & \\
\hline \multirow{2}{*}{$\alpha_{2}$} & $6 \leq t<10$ & \multirow{2}{*}{10.5} \\
\cline { 2 - 2 } & $20 \leq t<22$ & 14.5 \\
\hline$\alpha_{3}$ & $10 \leq t<20$ & \multirow{2}{*}{} \\
\hline
\end{tabular}

\section{RESULTS AND DISCUSSION}

Despite of the remarkable efforts made in energy management domain, still a big room left for research in terms of reducing energy cost and preserving consumer comfort simultaneously. Using all results generated for NS, VOLL, MST, and AMST, performance was evaluated for community energy consumption, community energy cost, renewable energy source influence, PAR value, and waiting time parameters.

Fig. 3 illustrates energy consumption results of NS, VOLL, and MST instances for 50 houses during 24 hours. As clearly visible in Fig. 3(a), off-peak energy consumption was increased in all houses for VOLL and MST compared to NS due to load shifting. As shown in Fig. 3(b), load profiles of community during average-peak hours varied closely in all three instances due lack of flexibility arise with consumption thresholds and tariffs. Peak energy consumption profiles of all houses are shown in Fig. 3(c) and clearly indicates the significant load reduction of MST compared to VOLL and NS. Although VOLL reduced peak load, it resulted 3.11\% load lost from total load. Total energy consumption of the community and average consumption per house are presented in Fig. 3(d). Total peak load reduction obtained by VOLL and MST are $180 \mathrm{kWh}$ and $264 \mathrm{kWh}$ respectively. In community perspective, MST reduced peak load by $7.89 \%$ compared to VOLL scheduling without any lost load. Corresponding average standard deviation values for average energy consumption are given below in Table II.

Table II. Standard deviation values for average energy consumption in residential community for NS, VOLL, MST scheduling

\begin{tabular}{|l|l|l|l|}
\hline & NS & VOLL & MST \\
\hline Off-peak & 1.62 & 2.19 & 2.46 \\
\hline Average-peak & 2.01 & 2.21 & 2.03 \\
\hline Peak & 5.11 & 4.52 & 4.30 \\
\hline
\end{tabular}



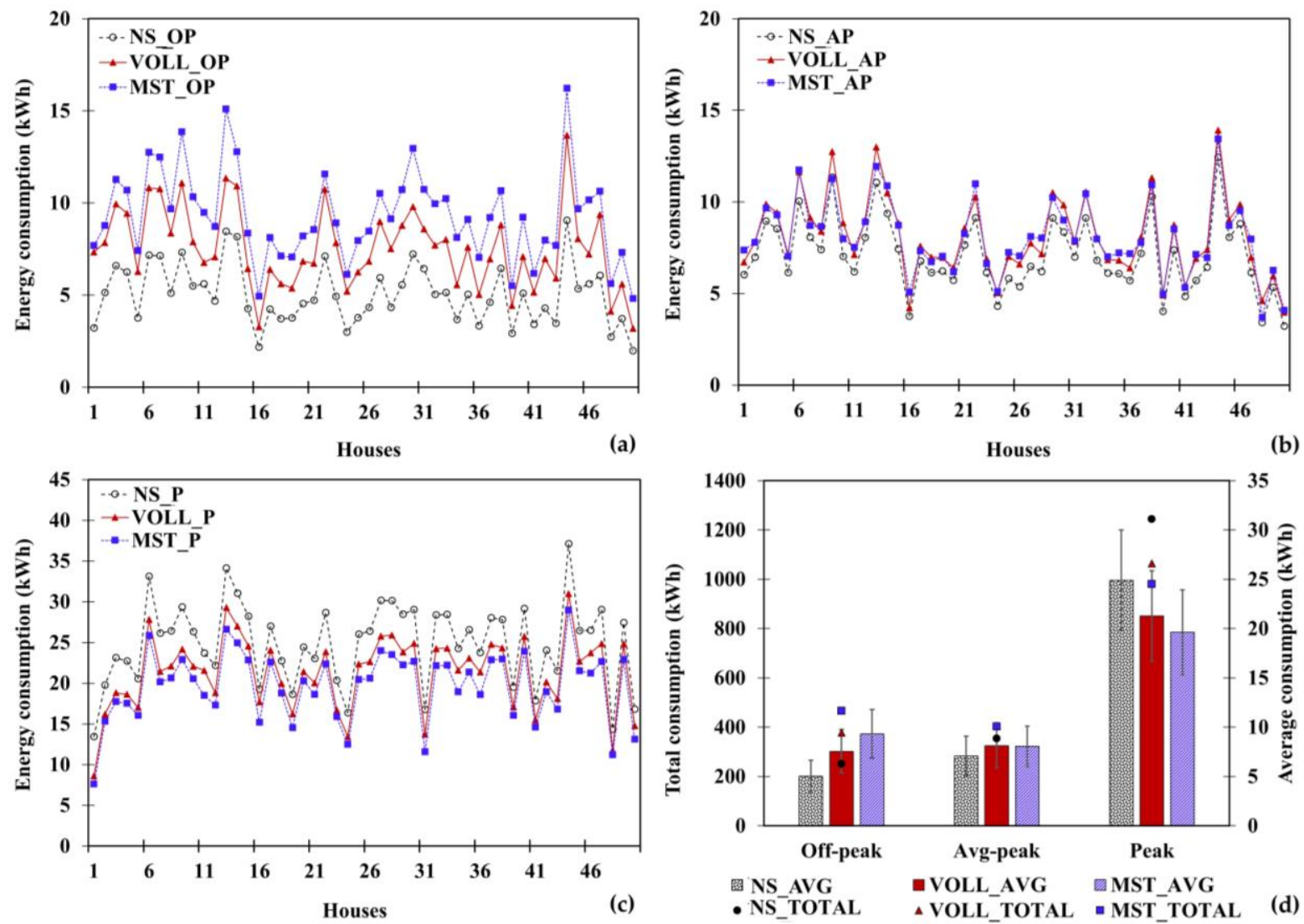

Fig. 3. Energy consumption of residential community. (a) Off-peak energy consumption (kWh); (b) Average-peak energy consumption (kWh); (c) Peak energy consumption (kWh); (d) Total and average energy consumption ((kWh) for each demand phase.
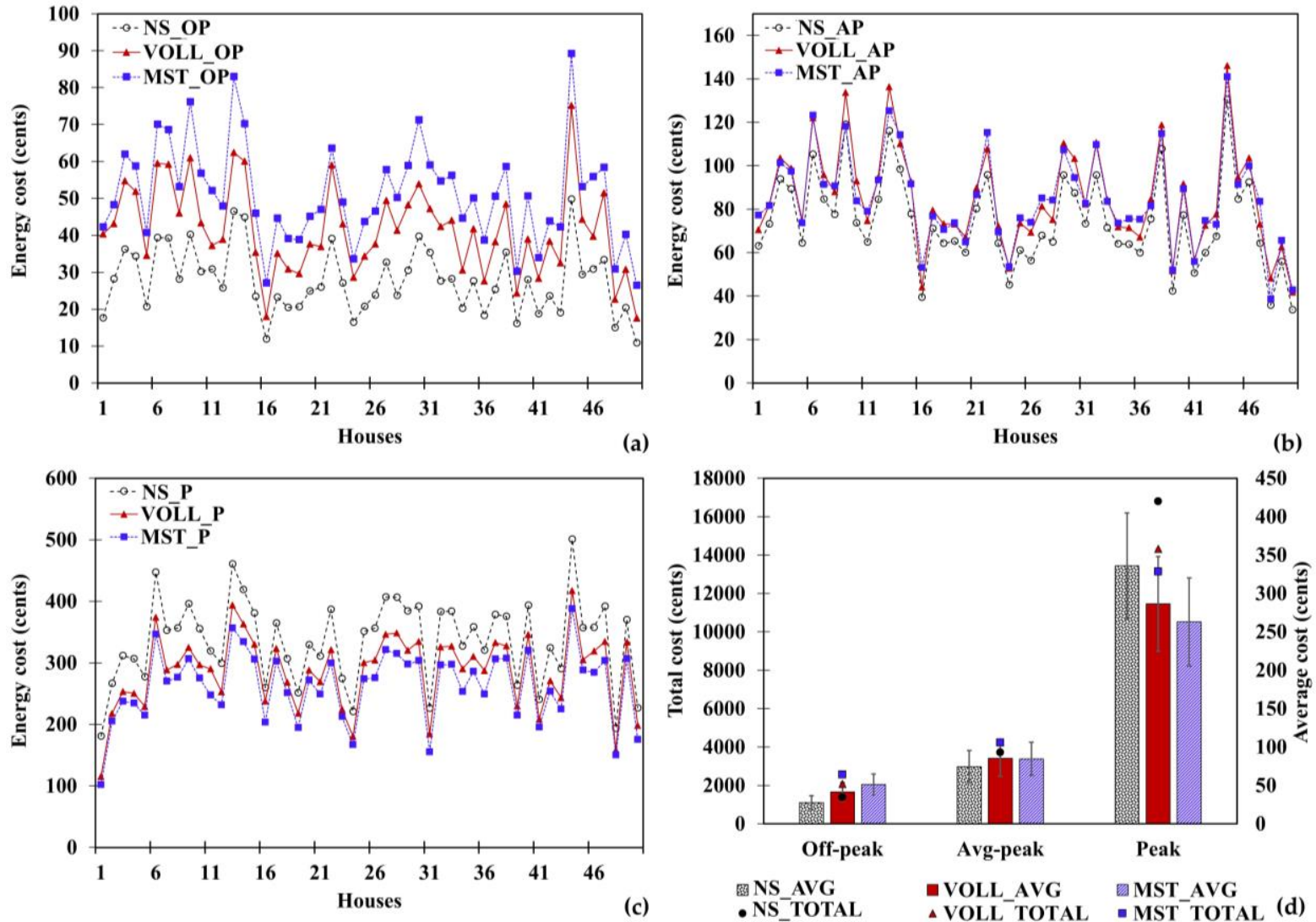

Fig. 4. Energy cost of residential community. (a) Off-peak energy cost (cents); (b) Average-peak energy cost (cents);

(c) Peak energy cost (cents); (d) Total and average energy cost (cents) for each demand phase. 
Community cost profiles for NS, VOLL, MST instances are presented in Fig. 4. All cost profiles correlate with consumption profiles in Fig. 3. Monetary value of energy consumed by each house during off-peak, average-peak, and peak hours are illustrated in Fig. 4(a), 4(b), and 4(c) respectively. It is clear from the results that MST reduced cost on energy than VOLL scheduling without introducing any lost load. Total and average cost profiles for the community during each demand phase are presented in Fig. 4(d). Total cost reduction achieved by VOLL and MST are 1214.5 cents and 1962.5 cents respectively. Average cost on energy during the day is reported as 438 cents, 413 cents, and 398 cents for NS, VOLL, and MST and corresponding standard deviations are given below in Table III.

Table III. Standard deviation values for average energy cost in residential community for NS, VOLL, MST scheduling

\begin{tabular}{|l|l|l|l|}
\hline & NS & VOLL & MST \\
\hline Off-peak & 8.90 & 12.09 & 13.57 \\
\hline Average-peak & 21.08 & 23.26 & 21.37 \\
\hline Peak & 68.98 & 61.68 & 57.61 \\
\hline
\end{tabular}

AMST instance integrates ESS and PV panels as alternative energy sources. Fig. 5 illustrates energy consumption profile of each house corresponding to energy source. AMST consumption profiles were compared only with proposed MST, since MST achieved superior performance over NS and VOLL with only grid electricity.

Noteworthy that PV energy is not aggregated during off-peak and average peak hours, since direct sunlight is not available during those time intervals. According to Fig. 5(a) and 5(b) AMST considerably reduced grid energy consumption during off-peak and average-peak hours, owing to ESS integration. Although Fig. 5(c) shows that cumulative peak load consumption of AMST is higher than MST, in all houses grid energy consumption is reduced owing to integration of PV panels and ESS. In other words, ESS and PV panels acted as alternative energy sources that take over peak load burden on grid. This phenomena improved consumer comfort by assuring minimal waiting time during peak hours. Fig. 5(d) depicts total and average energy consumption of the community with respect to energy sources. As depicted, in all demand phases, grid energy consumption of AMST is considerably less than MST. Since AMST outperforms MST, it is evident from the results that AMST certainly performs better than VOLL. Table IV presents standard deviations for average energy consumption with respect to energy source.

Table IV. Standard deviation values for average energy consumption in residential community for MST and AMST with respect to energy source

\begin{tabular}{|l|l|l|l|l|}
\hline & MST & AMST_GRID & AMST_PV & AMST_ESS \\
\hline Off-peak & 2.46 & 1.69 & - & 0.58 \\
\hline Average-peak & 2.03 & 1.96 & - & 0.43 \\
\hline Peak & 4.30 & 5.05 & 1.10 & 0.88 \\
\hline
\end{tabular}
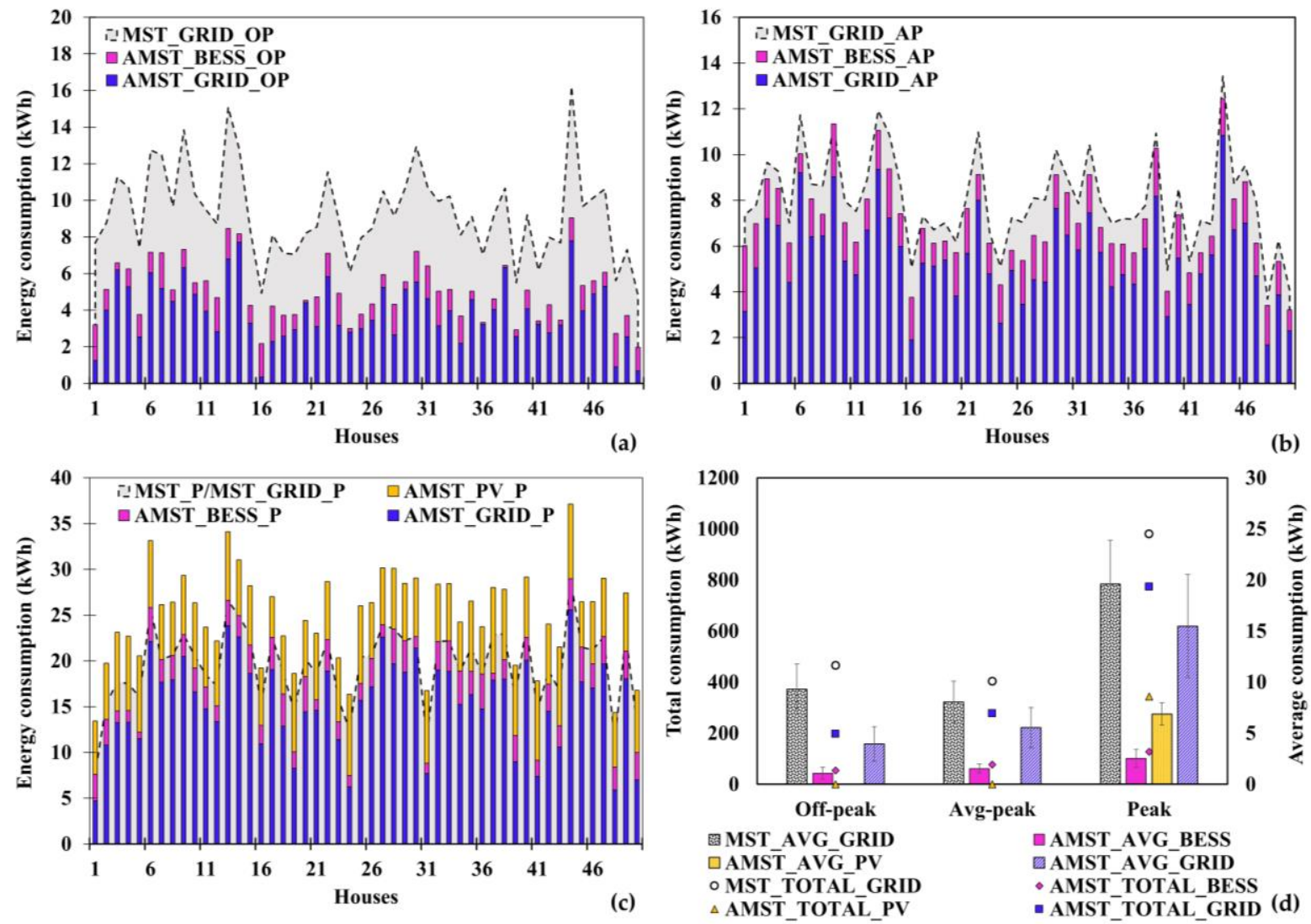

Fig. 5. Energy consumption of residential community with respect to energy source. (a) Off-peak energy consumption (kWh); (b) Average-peak energy consumption (kWh); (c) Peak energy consumption (kWh); (d) Total and average energy consumption ((kWh) for each demand phase 

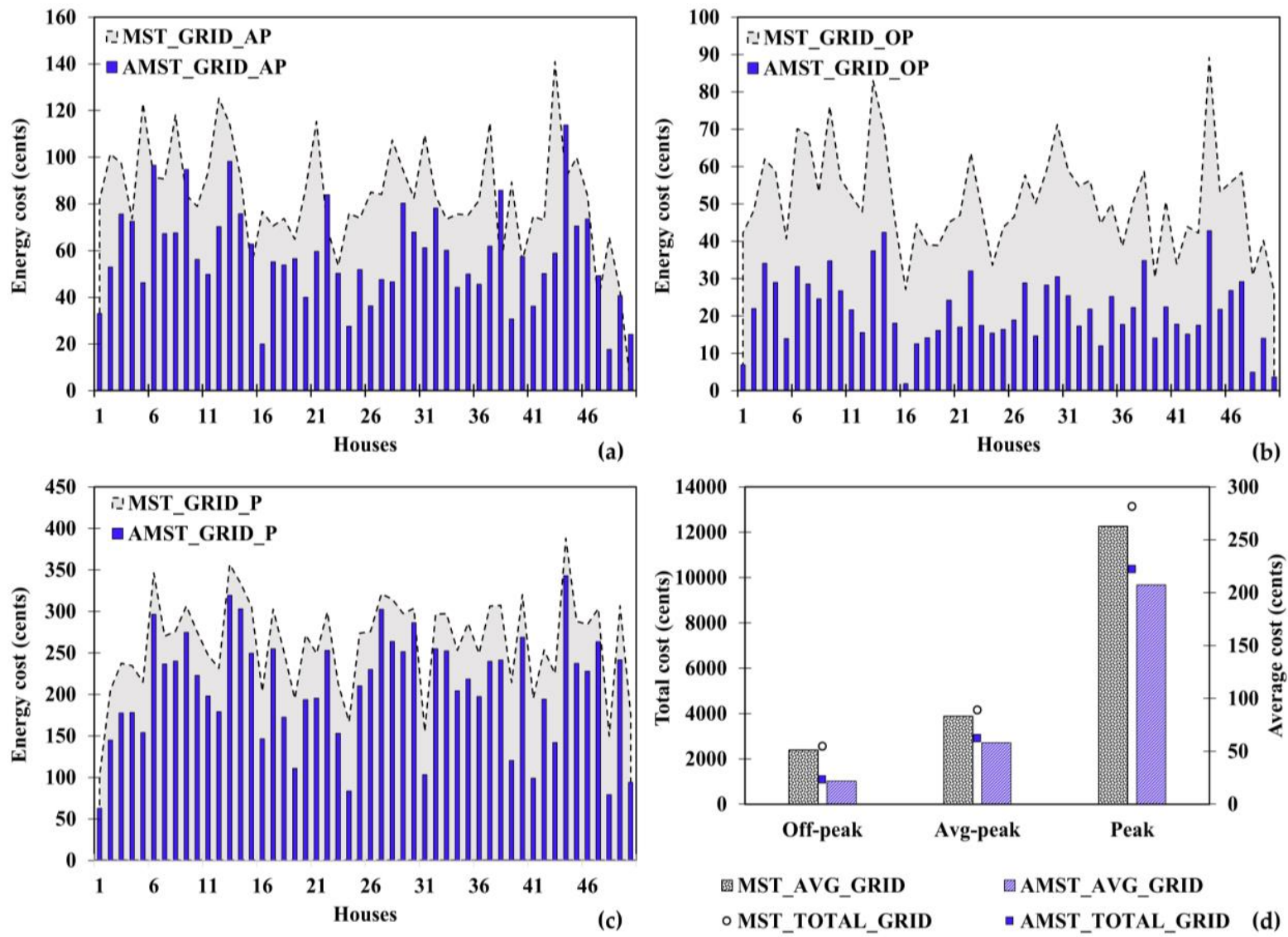

Fig. 6. Grid energy cost of residential community for MST and AMST. (a) Off-peak energy cost (cents); (b) Average-peak energy cost (cents); (c) Peak energy cost (cents); (d) Total and average energy cost (cents) for each demand phase.

Cost profiles of all houses in the community for grid energy utilization with MST and AMST DR are shown in Fig. 6. As clearly revealed in Fig. 6 (a), 6(b), and 6(c), reduction of grid energy utilization has replicated in all corresponding cost profiles. In community aspect, total energy cost for grid electricity was reduced by 5490 cents and average cost per house was reduced by 109 cents in AMST scheduling and distributed as in Fig. 6(d). Total cost reduction achieved by AMST with respect to NS, VOLL, and MST are given in Table V.
PAR value is the ratio between peak demand and average demand. Fig. 7(a) demonstrates PAR variation throughout the day for the whole community. PAR curve without any DR program (NS) has the steepest curve and several significant peaks. Although all other mechanisms have flattened the PAR curve to a certain extent, AMST has obtained the most flattened curve without any significant peaks. Hence, it is clear from the results that proposed MST and AMST manage grid peak demand without forming new peaks.
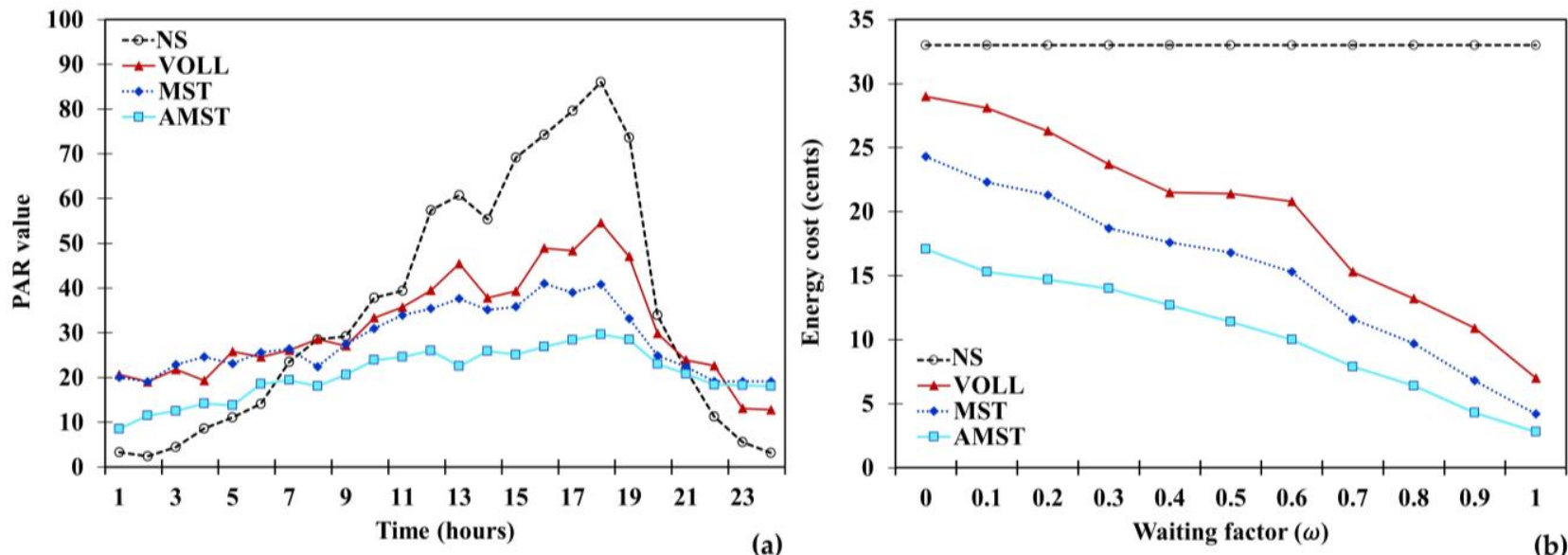

Fig. 7. PAR curve and waiting factor variation. (a) PAR value variation during the day for the community; (b) Correlation between waiting factor and cost reduction 
Table V. Cost reduction percentage achieved by AMST with respect to NS, VOLL, and MST

\begin{tabular}{|l|l|l|l|l|}
\hline \multirow{2}{*}{$\begin{array}{c}\text { AMST } \\
\text { against }\end{array}$} & \multicolumn{4}{|c|}{ Cost reduction (\%) } \\
\cline { 2 - 5 } & $\alpha_{1}$ & \multicolumn{1}{|c|}{$\alpha_{2}$} & \multicolumn{1}{c|}{$\alpha_{3}$} & $\alpha_{1}+\alpha_{2}+\alpha_{3}$ \\
\hline NS & 21.44 & 21.70 & 38.21 & 34.40 \\
\hline VOLL & 47.79 & 31.71 & 27.57 & 30.45 \\
\hline MST & 57.65 & 31.28 & 21.08 & 27.94 \\
\hline
\end{tabular}

Generally, DR programs compromise consumer comfort due to load shifting. Nevertheless, proposed work quantitatively minimizes waiting time to preserve maximum consumer convenience. Aggregating PV panels and ESS along with consumer defined constants contributed towards this goal. The objective function optimizes waiting factor and waiting time is calculated accordingly. The simulated scenario considered $\sigma_{1}=\sigma_{2}=0.5$. Cost variation with waiting factor is illustrated in Fig. 7(b). In the instance without any DR program, waiting factor did not influence energy cost, since operations are immediate. In VOLL, MST, and AMST instances energy cost was reduced with increasing waiting factor. However, as illustrated AMST has the optimal cost reduction with respect to $\omega_{f}$. Worthy to note VOLL scheduling obtained afore stated cost reduction with a small portion of lost load.

\section{DISCUSSION}

Although uncertainty of consumer patterns increase the complexity of DR programs, considering the uncertain nature of user behaviors is crucial for developing any successful DR program. Optimizing energy utilization to reduce monetary cost, while preserving consumer convenience is the utmost goal of any DR program. Nevertheless, this goal is tedious to achieve in real-world context due to the inverse correlation between cost and comfort. Many works reported in literature [13, 19-22] including our previous works $[15,16]$ successfully optimized electricity cost. However, these works did not focus on optimizing user convenience simultaneously and a majority of these works aimed to optimize energy consumption patterns of a single house, which mitigates the feasibility of realistic implementation. In order to address these challenges, herein we proposed a price driven DR program that optimizes energy utilization of a residential community, while independently preserving consumer convenience at each household.

Proposed MST algorithm was developed considering the concepts of LST algorithm. LST algorithm is less complex and widely used for real-time scenarios. Owing to dynamic priority driven nature of LST, proposed MST does not require a priori task information. [23]. In general, LST is well suited for preemptive tasks scheduling. Even though all tasks in a household are not preemptive, proposed MST alleviated this disadvantage by defining all appliances as state machines. Accordingly, non-preemptive tasks that get interrupted simply changes the state from active to sleep without terminating task operation. Performance of MST and AMST were compared with NS and VOLL, where VOLL is another DR program based on dynamic priority.

A shown in Fig. 3, both VOLL and MST disseminated energy demand across three demand phases. Although peak load demand was reduced in both VOLL and MST, unlike MST, VOLL achieved peak reduction with a portion of lost load that correlates with task priority. This causes lack of consumer satisfaction and was successfully addressed in proposed MST. Fig. 4 revealed that both VOLL and MST reduced electricity cost. VOLL is a price driven DR based on 2-tier IBR. During peak hours MST obtained the optimal cost, whereas VOLL optimized the cost to a certain extent by terminating low priority tasks. Hence, performance gains of MST can be explained as positive outcomes of introduced consumption thresholds and priority constants on cost and comfort. Fig. 5 and Fig. 6 presents consumption and cost profiles for MST scheduling after aggregating PV panels and ESS. As expected, these integrations significantly improved grid energy utilization and monetary expense on grid electricity. Hence, notable performance improvements of AMST can be explained as a consolidated result of aggregating alternative energy sources and afore stated characteristics of MST. PAR curve flattening in Fig. 7(a) achieved by MST and AMST is another remarkable benefit gained through consumption thresholds. These thresholds assure load dissemination among load phases without new peak formation. Cost reduction with waiting time is significant in proposed work as shown in Fig. 7(b), since the objective function quantitatively evaluates both cost and comfort. Users can determine maximum desired waiting time and priorities for cost and comfort. Owing to these characteristics, MST and AMST achieved superior performance over existing VOLL scheduling in terms of peak load shifting, cost reduction, grid energy utilization, PAR value reduction, and consumer convenience.

\section{CONCLUSIONS}

A ToU based energy management scheme based on LST algorithm was proposed for a residential community consists of 50 houses as a DR solution with less complexity and faster execution. Proposed MST scheduling was executed for 24 hours. The utmost objective of the work was to minimize electricity cost with aid of load shifting and alternative energy sources, while preserving consumer convenience. Propose work addressed uncertainty of consumer behaviors to mimic realistic operation. Underlying LST concepts assured that proposed MST is less complex and suitable for real-time scenarios. Further, consumption thresholds in MST ensured optimal load dissemination without new peak formation. Unlike other DR programs, priority constants defined in objective function simultaneously addressed cost and convenience parameters quantitatively. Moreover, optimal load dissemination flattened the PAR curve maintaining the balance between load demand and supply. Results revealed that proposed work successfully obtained minimal cost with minimal peak load compared to VOLL scheduling, which is an existing price driven DR program based on dynamic priority. Hence, results evidently claim that proposed MST and AMST works improve community energy utilization with preserved consumer satisfaction. Thus, proposed work will be a promising DR program that fits for futuristic smart communities.

\section{ACKNOWLEDGEMENT}

This work was supported by the National Research Foundation of 
Korea(NRF) grant funded by the Korea government(MSIT) (No. 2019R1F1A1042721) and BK21 Plus project (SW Human Resource Development Program for Supporting Smart Life) funded by the Ministry of Education, School of Computer Science and Engineering, Kyungpook National University, Korea (21A20131600005).

\section{REFERENCES}

1. A.-H. Mohsenian-Rad, A. Leon-Garcia, Optimal residential load control with price prediction in real-time electricity pricing environments, IEEE Trans. Smart Grid, 1 (2010) 120-133.

2. P. Charoen, M. Sioutis, S. Javaid, C. Charoenlarpnopparut, Y. Lim, Y. Tan, User-Centric Consumption Scheduling and Fair Billing Mechanism in Demand-Side Management, Energies, 12 (2019) 156.

3. J.A. Clarke, C.M. Johnstone, N.J. Kelly, P.A. Strachan, P. Tuohy, The role of built environment energy efficiency in a sustainable UK energy economy, Energy Policy, 36 (2008) 4605-4609.

4. B. Yuce, Y. Rezgui, M. Mourshed, ANN-GA smart appliance scheduling for optimised energy management in the domestic sector, Energy and Buildings, 111 (2016) 311-325.

5. Y. Liu, C. Yuen, S. Huang, N.U. Hassan, X. Wang, S. Xie, Peak-to-average ratio constrained demand-side management with consumer's preference in residential smart grid, IEEE Journal of Selected Topics in Signal Processing, 8 (2014) 1084-1097.

6. A.S. Awad, T.H. El-Fouly, M.M. Salama, Optimal ESS allocation for load management application, IEEE Transactions on Power systems, 30 (2015) 327-336.

7. C.P. Mediwaththe, E.R. Stephens, D.B. Smith, A. Mahanti, A dynamic game for electricity load management in neighborhood area networks, IEEE Transactions on Smart Grid, 7 (2016) 1329-1336.

8. M. Muratori, G. Rizzoni, Residential demand response: Dynamic energy management and time-varying electricity pricing, IEEE Transactions on Power systems, 31 (2016) 1108-1117.

9. M.F. Haniff, H. Selamat, R. Yusof, S. Buyamin, F.S. Ismail, Review of HVAC scheduling techniques for buildings towards energy-efficient and cost-effective operations, Renewable and Sustainable Energy Reviews, 27 (2013) 94-103.

10. D. Setlhaolo, X. Xia, J. Zhang, Optimal scheduling of household appliances for demand response, Electric power systems research, 116 (2014) 24-28.

11. C.O. Adika, L. Wang, Autonomous appliance scheduling for household energy management, IEEE Transactions on Smart Grid, 5 (2014) 673-682.

12. X. Chen, T. Wei, S. Hu, Uncertainty-aware household appliance scheduling considering dynamic electricity pricing in smart home, IEEE Transactions on Smart Grid, 4 (2013) 932-941.

13. M.A.A. Pedrasa, T.D. Spooner, I.F. MacGill, Coordinated scheduling of residential distributed energy resources to optimize smart home energy services, IEEE Transactions on Smart Grid, 1 (2010) 134-143.

14. R.M. Shukla, P. Kansakar, A. Munir, A Neural Network-based Appliance Scheduling Methodology for Smart Homes and Buildings with Multiple Power Sources, in: Nanoelectronic and Information Systems (iNIS), 2016 IEEE International Symposium on, IEEE, 2016, pp. 166-171.

15. M. Khan, B.N. Silva, K. Han, Internet of Things Based Energy Aware Smart Home Control System, IEEE Access, 4 (2016) 7556-7566.

16. B.N. Silva, M. Khan, K. Han, Load Balancing Integrated Least Slack Time-Based Appliance Scheduling for Smart Home Energy Management, Sensors, 18 (2018) 685.

17. M. Khan, B.N. Silva, C. Jung, K. Han, A context-Aware Smart Home Control System based on ZigBee Sensor Network, KSII Transactions on Internet and Information Systems, 11 (2017) 1057-1069.

18. B.N. Silva, K. Han, Mutation operator integrated ant colony optimization based domestic appliance scheduling for lucrative demand side management, Future generation computer systems, 100 (2019) 557-568.

19. Z. Zhao, W.C. Lee, Y. Shin, K.-B. Song, An optimal power scheduling method for demand response in home energy management system, IEEE Transactions on Smart Grid, 4 (2013) 1391-1400

20. M. Rastegar, M. Fotuhi-Firuzabad, H. Zareipour, Home energy management incorporating operational priority of appliances, International Journal of Electrical Power \& Energy Systems, 74 (2016) 286-292.

21. N. Kumaraguruparan, H. Sivaramakrishnan, S.S. Sapatnekar, Residential task scheduling under dynamic pricing using the multiple knapsack method, (2012).
22. T. Logenthiran, D. Srinivasan, T.Z. Shun, Demand side management in smart grid using heuristic optimization, IEEE Transactions on Smart Grid, 3 (2012) 1244-1252.

23. S. Park, J.-H. Kim, G. Fox, Effective real-time scheduling algorithm for cyber physical systems society, Future generation computer systems, 32 (2014) 253-259.

\section{AUTHORS PROFILE}

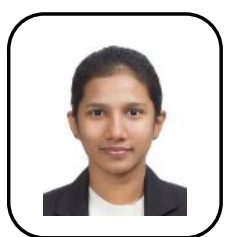

Bhagya Nathali Silva received the B.S. and M.S degrees in Information Technology from Sri Lanka Institute of Information Technology, Sri Lanka, in 2011 and 2015 respectively. She received the Ph.D in Computer Science and Engineering from Kyungpook National University, Korea in 2018 and currently working as a Contract Professor at Kyungpook National University, Korea. She served as a reviewer in numerous reputed journals including Future Generation Computer Systems, Sustainable Cities and Society, Sensors, IEEE Access, etc. Her area of expertise includes architecture designing for Internet of Things, Machine-to Machine Communication, Cyber Physical Systems, and Communication Protocols, etc.

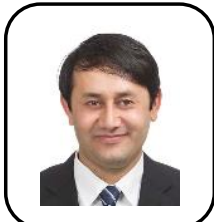

Murad Khan received the B.S. degree in Computer Science from the University of Peshawar Pakistan in 2008. He completed the Master and Ph.D. degrees in Computer Science and Engineering from School of Computer Science and Engineering at Kyungpook National University, Daegu, Korea and currently serving as a Korea Research Fellow sponsored by Korea Research Foundation. He also served as a TPC member in world reputed conferences and as a reviewer in numerous journals such as Future Generation Systems (Elsevier), IEEE Access, Sustainable Cities and Society Journal, etc. He is a member of ACM and IEEE, CRC press, etc. His area of expertise includes vertical handover management in heterogeneous wireless networks, architecture designing for the Internet of Things, Communication Protocols designing for smart cities and homes, and Big Data Analytics.

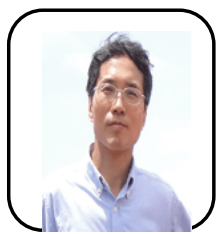

Kijun Han received the B.S. degree in electrical engineering from Seoul National University, Korea, in 1979 and the M.S. degree in electrical engineering from the KAIST, Korea, in 1981 and the M.S. and Ph.D. degrees in computer engineering from the University of Arizona, in 1985 and 1987, respectively. He has been a professor of School of Computer Science and Engineering at the Kyungpook National University, Korea since 1988. His area of expertise include Computer networks, Wireless Sensor Networks, Communication Protocols, Internet of Things, etc. 